\title{
Determinantes de la competitividad regional en el Distrito Federal: un análisis del índice de competitividad estatal (2008-2012)
}

\author{
Emma Frida Galicia Haro* \\ Ana Lilia Coria Páez* \\ Alexander Galicia Palacios*
}

\section{Resumen}

Entre los problemas del crecimiento económico se presenta la interrogante sobre por qué existen territorios que pueden mantener tasas constantes de crecimiento con mejoría en las condiciones de vida de sus ciudadanos y otras no. Esto ha llevado en los últimos años al auge del estudio de la competitividad y en particular de la competitividad regional. El objetivo de esta investigación es identificar los indicadores que han favorecido la competitividad del Distrito Federal, en el contexto de las diversas teorías del crecimiento económico, a través del índice de competitividad estatal (ice) elaborado por el imco en el periodo 2008-2012. Se concluye que los subíndices sectores precursores, sociedad incluyente, preparada y sana, aprovechamiento de las relaciones internacionales e innovación contienen indicadores que impulsan la competitividad del Distrito Federal, sustentados por las teorías que promueven los modelos del desarrollo endógeno.
Abstract

The question presents itself between the problems of economic growth because there are territories that can be kept constant rates of growth with improvement in the living conditions of their citizens, and others do not. This has led in recent years to the rise of the study of competitiveness and in particular of regional competitiveness. The objective of this research is to identify indicators which have enhanced the competitiveness of the Federal District, in the context of the various theories of economic growth, through State competitiveness index (ice) prepared by the imco in the period 2008-2012. It is concluded that subscripts pioneering sectors, inclusive, prepared and healthy society taking advantage of international relations andinnovation containindicators thatdrive the competitiveness of the Federal District supported by theories that promote the endogenous development model.

Palabras clave: competitividad regional, desarrollo regional, crecimiento económico, desarrollo endógeno, medición.

Key words: regional competitiveness, regional development, economic growth, endogenous development, measurement.

\footnotetext{
*Instituto Politécnico Nacional, esca Tepepan Correos electrónicos: fgalicia@ipn mx; copa7013@
} hotmail com; alex_finster@hotmail.com 


\section{Antecedentes}

Desde finales del siglo pasado el interés por identificar los procesos que mejoran el crecimiento económico de los países ha impulsado que un gran número de estudiosos aborden el tema de la competitividad y con él su definición, identificación de causas y medición de sus efectos. Si bien existen autores que identifican a la competitividad como un tema que aparece desde el siglo xvi, dichos enfoques iniciales y vigentes por varios siglos asocian el tema básicamente con el estímulo que brinda a un país el desarrollar su comercio exterior y el análisis se centra en los elementos que influyen en éste Estas ideas iniciales se atribuyen a los mercantilistas, que centraban la creación de riqueza en la acumulación de oro y joyas, y aun cuando la teoría evolucionó postulando que los países más competitivos eran aquellos que podían mantener un saldo positivo en su intercambio comercial con el exterior (Mun, 1978), su postulado inicial proponía el mantenimiento de un equilibrio permanente en el balance con el exterior con una cierta protección para éste (Blaug, 1985)

La propuesta anterior fue criticada por los representantes de la economía clásica, iniciada por Adam Smith (Cho y Moon, 2002), que postulaba el libre comercio internacional, considerado como el mecanismo que favorecería la eficiencia en la producción con bajos costos, generando además una clara división internacional del trabajo, en un contexto de mínima intervención del Estado, preservando la libre competencia, lo que traería en consecuencia que la nación produciría los bienes de más bajo costo obteniendo lo que conocemos como ventaja absoluta. Dentro de esta misma corriente, David Ricardo, a partir de los postulados de Smith propuso la teoría de la ventaja comparativa, al incorporar los costos relativos en el uso más eficiente de los recursos (Cho y Moon, 2002)

Estos enfoques en esencia permanecieron vigentes y fueron ampliamente aplicados durante siglos Su pérdida de vigencia vendría derivada de la emergencia de las grandes transformaciones observadas en el siglo xx, las grandes crisis económicas y el fenómeno de la globalización Durante la primera mitad del siglo xx el surgimiento de la crisis de 1929 evidencióla incapacidad de respuesta del mercado frente a un desequilibrio que no mejoraba como respuestaa las medidas ortodoxas quela teoría neoclásicaestablecía Lateoría 
keynesiana recomendaba, frente a la imposibilidad de que el mercado garantizara el pleno empleo, alentar el papel activo del gobierno como un estabilizador de las deficiencias del mercado.

Keynes (2013) incorpora la teoría de la demanda agregada y sus componentes básicos: consumo, inversión, gasto público y exportaciones netas, abriendo un ciclo de pensadores que identificaban nuevos componentes que influenciaban la competitividad de las naciones mediante su impacto en el consumo y la inversión; así que para Thurow (citado por Staskevičiūtė y Tamošiūnienè, 2010) la creación de políticas económicas gubernamentales de impulso es fundamental para el progreso, y para Schumpeter lo importante es poner especial énfasis en el papel de los empresarios y en la idea de que el desarrollo de una nación se da por cambios y que éstos son impulsados por la innovación y el desarrollo tecnológico

Solow comparte esta idea y hace un análisis de los factores que impulsan el crecimiento, destacando la innovación científica y tecnológica a partir de unaidea que consolidarála creación de nuevos enfoques de la competitividad al señalar que el rendimiento de la inversión y por lo tanto la tasa de crecimiento del stock de capital per cápita disminuye a medida que el stock crece (Staskevičiūtè y Tamošiūnienè, 2010).

A partir de la década de los ochenta, dominada por crisis recurrentes a nivel mundial y la ampliación de la desigualdad mundial entre las naciones ricas y las pobres, las ideas neoclásicas resurgieron desde los principales organismos internacionales en forma de la revalorización del mercado como el garante de la estabilidad y de reducción de la brecha entre ricos y pobres, una reducción de la injerencia estatal en el diseño de políticas y una gran apertura hacia el exterior con la finalidad de alcanzar el mercado global (Thwaites Rey, 2010)

El fracaso de estos enfoques derivó hacia finales de los años ochenta en el surgimiento de un grupo de propuestas englobadas en el término "modelos de crecimiento endógeno" (mce) La base de su desarrollo se encuentra en hacer dependiente a la tasa de crecimiento económico de tres factores: el capital físico, el capital humano y el nivel de progreso técnico Lo que tiene como efecto en el caso del capital humano que los rendimientos están asociados a su crecimiento, a la calidad del aprendizaje en la práctica, y a considerar como un factor productivo adicional el monto de conocimiento acumulado 
Por lo tanto en este esquema son determinantes la educación formal, la investigación y el desarrollo (Mattos, 1999)

A estos desarrollos se incorporan las aportaciones de Porter (2015), que enfatiza el papel de la empresa como responsable de la productividad y por lo tanto de la competitividad; asimismo asegura que no existe competitividad sin productividad, siendo ésta la base del éxito de los países, e incorpora el papel del Estado como garante para crear un ambiente que ayude a las empresas a crecer e innovar más rápido que sus competidores extranjeros

Por último, en los primeros años del siglo xxi a estos nuevos enfoques se ha agregado el elemento bienestar social, condicionando la existencia de la competitividad a la existencia y recuperación de altos niveles de vida (Huggins, Izushi, y Thompson, 2013)

\section{Competitividad regional}

Las teorías que dan origen al concepto de la competitividad han ido evolucionando a lo largo de los años y esto dificulta la obtención de una definición única y consensuada del término. Más aún para la definición de competitividad regional, ya que su ubicación en el ámbito macroeconómico es todavía más controvertida Aplicar la idea de lo macroeconómico linealmente a lo regional no es válido por la distancia cuantitativa y cualitativa existente entre estas dos realidades Sin embargo, el estudio de las regiones ha ido tomando relevancia en los años recientes a partir de la experiencia de que son las regiones un centro de desarrollo económico y un adecuado lugar para lograr la organización empresarial (Maleki, 2014)

Será necesario tomar en cuenta, para generar una definición de la competitividad regional, que existe una problemática a superar ya que, en primer lugar no cabe duda que una región no es un país en la medida en que no es totalmente independiente en la elaboración de políticas públicas, en particular en algunas que impactan directamente los sustentos de la competitividad: inflación, tipo de cambio, tasa de interés, regulaciones comerciales internacionales, entre otras Para Krugman (1994) resulta que la competitividad puede tornarse una obsesión que llega al punto de equipar el desempeño de los países o regiones con el de una empresa, lo cual no es aceptable dado que como expresa Krugman, una empresa que no es competitiva si no mejora deja de 
existir, en tanto que una nación no puede despedir a sus ciudadanos y cerrar sus operaciones

De acuerdocon Huggins, Izushi y Thompson (2013), la conceptualización de la competitividad regional al estar inmersa en este controvertido debate sobre su definición, va tomando forma a través de los elementos constitutivos de los modelos de crecimiento, en paralelo con los temas relativos a los nuevos componentes que explican la competitividad. Proponen como definición de competitividad regional "la capacidad de las regiones para lograr crecimiento económico en relación con otras regiones en una fase similar de desarrollo económico global, dentro de su propia nación o bloque continental", en tanto que Stoper (citado por Huggins, Izushi y Thompson, 2013) a finales de los años noventa señalaba que consistía en la capacidad de una región en particular para atraer y mantener empresas con cuotas de mercado estable o un aumento de una actividad mientras que permanecía o crecía el nivel de vida de los participantes

Alaproblemática de no contar con una definición consensuada del término se añade el problema de su medición, que presenta dificultades debido tanto a la ausencia de índices específicos de medición como a una gran variedad de propuestas Se observa que a nivel mundial existe literatura y propuestas de medición a nivel nacional que coinciden con las dificultades aquí descritas en cuanto a la definición del término y se subsana con la metodología de cálculo. Entre otros, se pueden mencionar los índices de competitividad nacional realizados por instituciones internacionales reconocidas: wef(World Economic Forum, 2014), imd (International Institute for Management Development, 2014) y en estudios regionales de la Unión Europea a partir de modelos econométricos (Melecký, 2011), así como referentes a la metodología de cálculo (Huggins, Izushi y Thompson, 2013) Las mediciones, si bien establecen metodologías robustas, se refieren en su mayoría a estudios transversales que no permiten apreciar el desempeño de la competitividad a lo largo del tiempo Por lo que la evidencia empírica que cuantifica la competitividad regional existe a nivel nacional y sólo como referencia entre países, sin poder identificar la eficiencia en la conducción de la política económica y sus resultados en la productividad o en el nivel de vida 


\section{Objetivo de la investigación}

Identificar los determinantes de la competitividad regional en el Distrito Federal por medio de un análisis del índice de competitividad estatal (ice) elaborado por el Instituto Mexicano para la Competitividad (imco) durante el periodo 2008-2012, con el fin de identificar cómo en determinada región se administran los recursos y capacidades a objeto de incrementar sostenidamente la productividad empresarial y el bienestar de su población

\section{Método de investigación}

Éstaes unainvestigación cualitativa con unalcance descriptivoque considera la revisión teórica de la competitividad regional mediante el análisis del contenido de las diversas teorías del crecimiento económico y del debate alrededor de la conceptualización de la competitividad regional

La investigación se inició con la síntesis de los elementos relativos a la competitividad incluidos en los postulados teóricos de las teorías más representativas del desarrollo económico y la controversia existente sobre el concepto de competitividad regional reunidos en una investigación documental A continuación se documentaron los hallazgos de investigaciones referentes a la medición de la competitividad regional de México, describiendo su método de cálculo y el resultado de la competitividad en el Distrito Federal Se seleccionó el ice del imco para obtener información sobre el desempeño cuantitativo de los 10 subíndices y 89 variables que lo integran en el periodo 2008-2012, se analizó el desempeño de los 10 subíndices para identificar los de desempeño superior al promedio nacional, se hizo el análisis de los indicadores que integran a los subíndices de buen desempeño para identificar a qué corriente teórica pertenecían, y finalmente se elaboró una síntesis de los posibles orígenes teóricos que guían las políticas locales a favor de la competitividad

\section{Análisis empírico: la medición de la competitividad del Distrito Federal}

El estudio de la competitividad regional en México se ha abordado desde diferentes perspectivas La Cámara de Diputados, por conducto del Centro 
de Estudios Sociales y de Opinión Pública desarrolló dos estudios: uno sobre los municipios de México (Centro de Estudios Sociales y de Opinión Pública, Cámara de Diputados — cesop—- 2008) y el otro sobre desarrollo regional y competitividad en México (cesop, 2012) En este último se compilan varios estudios sobre el desarrollo regional sin llegar a mediciones del mismo; la intención se concentró en el análisis cualitativo sobre el desempeño regional con el fin de proponer políticas a favor del desarrollo local. En tanto que en el primero se hace un comparativo de tres índices de competitividad regional en México para los años 2003-2007, el desarrollado por el imco, el del cide y el de la consultora Aregional Las mediciones, si bien tienen metodologías muy diferentes, presentan para el caso de la ciudad de México resultados similares En las tres mediciones la ciudad de México se ubica dentro de los primeros lugares del ranking $\left(6^{\circ}, 3^{\circ}\right.$ y $6^{\circ}$, respectivamente $)$

Sobrino (2010) parte del método diseñado por Ni Pengfei para estimar la competitividad de las ciudades de México; selecciona cinco variables: a) logaritmo del pib total en 2003 (pib); b) tasa de crecimiento del pib entre 1998 y 2003 (tcpib); c) logaritmo del pib por habitante en 2003 (pibph); d) tasa de ocupación promedio en el periodo (toc), y e) índice de calidad de vida (icv) La ciudad de México registró el tercer lugar bajo estas condiciones En tanto que para Cabrero, Orihuela y Ziccardi (2003) el cálculo se realiza al aplicar cuatro componentes para medir la competitividad de las ciudades de México entre 1998 y 2000; los cuales corresponden a: a) económico; b) sociodemográfico; c) urbano-espacial, yd)institucional. El resultado final es que la zona metropolitana de la ciudad de México queda en $4^{\circ}$ lugar en el índice promedio En el económico en $7^{\circ}$, en el institucional en $4^{\circ}$, en el urbano en el $7^{\circ} \mathrm{y}$ en el sociodemográfico cae hasta el lugar 33.

Todos estos estudios reflejan de diferentes formas la dificultad que implica valorar la competitividad regional en México, derivada de los temas de delimitación geográfica, heterogeneidad de los métodos de cálculo, seguimiento de la medición en el tiempo y diversidad de interpretación de los resultados

En el caso del imco, se define en su página web como:

Un centro de investigación aplicada basada en evidencia Nuestra misión institucional es serconsultores dela sociedad mexicana en materia de políticas públicas Somos unainstitución independiente, apartidista y sin fines de lucro, dedicada a elaborar propuestas viables 
para mejorar la capacidad de México para atraer y retener talento e inversiones (Instituto Mexicano para la Competitividad, 2015)

Su aportación al cálculo de la competitividad regional es difundir el índice de competitividad estatal con datos anuales y periodicidad diversa, sujeta a la obtención de datos, ya que la emisión del índice puede no coincidir con el último año o el anterior, como es el caso del último reporte, que aun cuando se denomina índice de competitividad estatal, 2014, los datos que tomó como base se refieren al año 2012. (Para los efectos de este trabajo se tomó el año real )

En sus notas metodológicas se hace una exhaustiva descripción de las fuentes de obtención de datos, su clasificación, su consistencia respecto a los de años diferentes, la valoración en una escala de 0 a 100 de los indicadores y se enuncia el modelo estadístico usado para su cálculo como un modelo de regresión lineal que establece una relación entre formación bruta de capital fijo por pea y talento (la proporción de la población mayor de 25 años con estudios superiores) con los 10 subíndices de competitividad, así como con el índice de competitividad general, validado mediante el estadístico pca (análisis de componentes principales) combinado con el alfa de Conbrach para su consistencia interna

Esteindicador define a la competitividad "como la capacidad de atraery retener talento e inversión" y se compone de 10 subíndices: 1 Sistema de derecho confiable y objetivo. 2. Manejo sustentable del medio ambiente. 3. Sociedad incluyente, preparada y sana 4 Sistema político estable y funcional 5 Gobiernos eficientes y eficaces. 6. Mercado laboral. 7. Economía estable. 8 Sectores precursores 9 Aprovechamiento de las relaciones internacionales, y 10 Innovación de los sectores económicos, desglosados en cantidades diferentes con un total de 89 indicadores (Instituto Mexicano para la Competitividad, 2015)

En la metodología empleada se definen ponderaciones que otorgan importancia a cada uno de ellos, como se muestra en el cuadro 1 


\section{Cuadro 1}

Ponderaciones de los componentes del ice del imco (\%)

\begin{tabular}{l|c}
\hline Subíndice & Peso \\
\hline Sistema de derecho confiable y objetivo & 10.1 \\
\hline Manejo sustentable del medio ambiente & 8.4 \\
\hline Sociedad incluyente, preparada y sana & 14.4 \\
\hline Sistema político estable y funcional & 5.4 \\
\hline Gobiernos eficientes y eficaces & 7.2 \\
\hline Mercado laboral & 8.9 \\
\hline Economía estable & 12.1 \\
\hline Sectores precursores & 12.6 \\
\hline Aprovechamiento de las relaciones internacionales & 8.6 \\
\hline Innovación de los sectores económicos & 12.3 \\
\hline Total & 100.0 \\
\hline
\end{tabular}

Fuente: elaboración propia con datos del ice (imco, 2015)

\section{Resultados}

Al elaborar las series para el periodo 2008-2012 se encontró que los mejores desempeños corresponden a los sectores precursores, sociedad incluyente preparada y sana, aprovechamiento de las relaciones internacionales e innovación en los sectores económicos (gráficas 1 al4):

\section{Gráfica 1}

Desempeño 2008-2012 del subíndice sectores precursores del ice

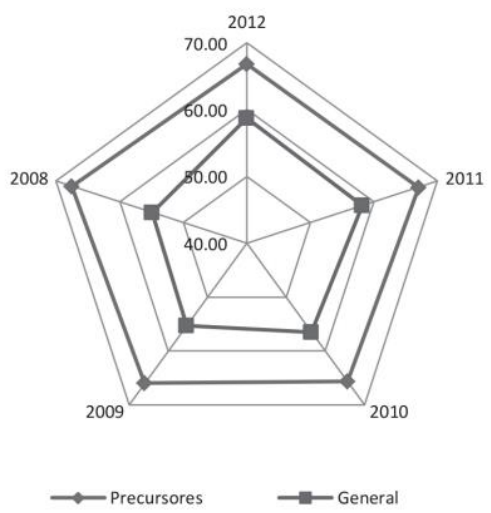

Fuente: elaboración propia con datos del ice (imco, 2015) 


\section{Gráfica 2}

Desempeño 2008-2012 del subíndice sociedad incluyente, preparadaysanadelice

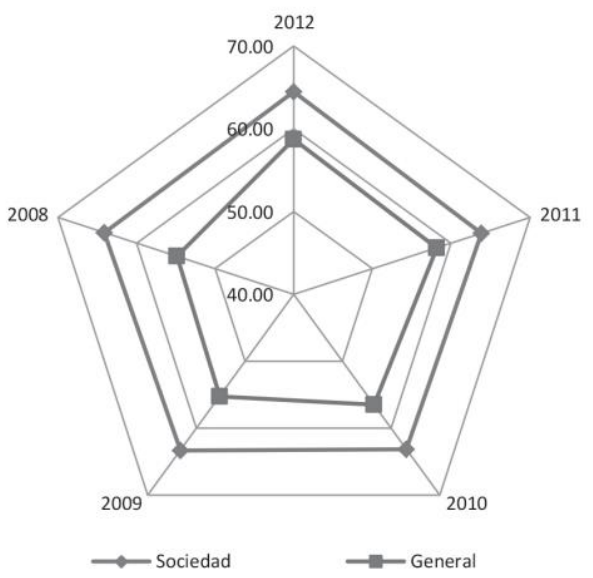

Fuente: elaboración propia con datos del ice (imco, 2015)

\section{Gráfica 3}

Desempeño 2008-2012 del subíndice aprovechamiento de las relaciones internacionales del ice

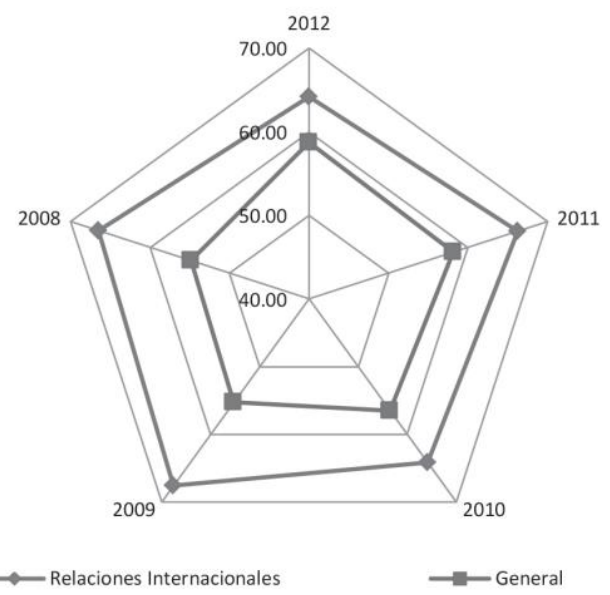

Fuente: elaboración propia con datos del ice (imco, 2015) 


\section{Gráfica 4}

Desempeño 2008-2012 del subíndice innovación del ice

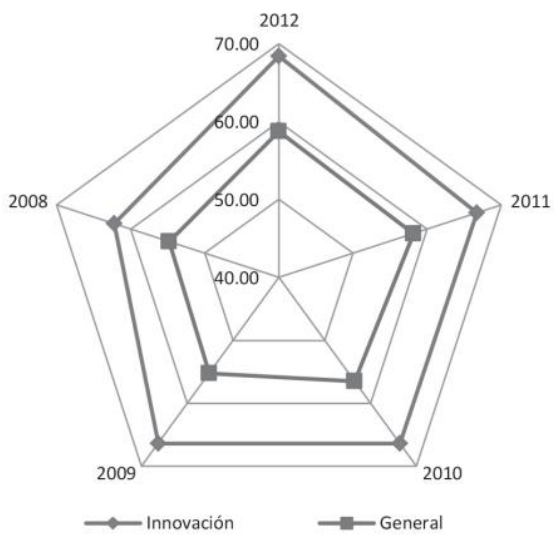

Fuente: elaboración propia con datos del ice (imco, 2015)

Con base en la ponderación adoptada por el imco, ya mencionada, el conjunto de los cuatro subíndices tienen una ponderación en el índice que en conjunto suma $47.8 \%$ del total de 10 subíndices, lo cual supone la influencia relevante de éstos en la ubicación del Distrito Federal como el primer lugar en el índice, con una valoración de 626 puntos sobre 100 posibles y una diferencia de 18 puntos respecto al promedio nacional $\mathrm{Al}$ analizar los indicadores que los conforman y compararlos con las propuestas teóricas mencionadas (cuadro 2) es posible observar que se concentran en aquellos que se ubican en las corrientes teóricas de los modelos de crecimiento endógeno (mce) y en menor medida por un cierto rasgo del keynesianismo 
Cuadro 2

Clasificación de los subíndices del ice en el contexto de las teorías del desarrollo económico

\begin{tabular}{|c|c|c|}
\hline Aspectos relevantes sobre la competitividad & Postulados teóricos & Subíndice \\
\hline $\begin{array}{l}\text { Superávit comercial } \\
\text { Protección del comercio }\end{array}$ & Mercantilismo & \\
\hline $\begin{array}{l}\text { Libre mercado } \\
\text { Costos bajos ventaja absoluta } \\
\text { Costos relativos ventaja comparativa }\end{array}$ & Clásicos & \\
\hline $\begin{array}{l}\text { Demanda agregada } \mathrm{C}+\mathrm{I}+\mathrm{GP}+\mathrm{Xn} \\
\text { Impacto del comercio exterior en el } \\
\text { consumo y la inversión } \\
\text { Intervención del Gobierno como } \\
\text { estabilizador }\end{array}$ & Keynes & $\begin{array}{l}\text { Aprovechamiento } \\
\text { de las relaciones } \\
\text { internacionales }\end{array}$ \\
\hline $\begin{array}{l}\text { Política económica efectiva } \\
\text { Papel de los empresarios } \\
\text { La innovación y el desarrollo tecnológico } \\
\text { La innovación científica y tecnológica }\end{array}$ & $\begin{array}{l}\text { Nuevos } \\
\text { componentes } \\
\text { Modelos de } \\
\text { desarrollo } \\
\text { endógeno mce } \\
\end{array}$ & Innovación \\
\hline \multirow{2}{*}{$\begin{array}{l}\text { Elevación del nivel de vida de los } \\
\text { ciudadanos }\end{array}$} & \multirow[t]{2}{*}{ El enfoque social } & Sectores precursores \\
\hline & & $\begin{array}{l}\text { Sociedad incluyente } \\
\text { preparada y sana }\end{array}$ \\
\hline
\end{tabular}

Fuente: elaboración propia con base en autores revisados y el ice del imco (2015)

Los rasgos característicos de los modelos de crecimiento endógeno (mce) hacen dependiente al incremento del capital de los factores capital físico, humano y conocimiento, como fuentes de crecimiento económico del país Al incorporar el conocimiento se da impulso al protagonismo de la educación, la innovación y el desarrollo, que lleva a poner en primer plano el desarrollo del capital humano tanto por su capacidad de generar i\&d como por la necesidad de proteger y elevar su nivel de vida Lo que coincide con los indicadores de los subíndices "innovación", "sectores precursores" y "sociedad incluyente, preparada y sana" que tienen como indicadores aspectos relacionados con esta corriente de pensamiento, como se puede observar en el cuadro 3

En segundo lugar se tiene la adopción de medidas derivadas de la teoría keynesiana, sin llegar a ser una propuesta ortodoxa ni cercana a un estatismo, adoptando la idea de valor aportado por el sector externo Dando prioridad a los ingresos del sector turismo Sin que la comparación sea totalmente vá- 


\section{Cuadro 3}

Clasificación de los indicadores por subíndices del icR en el contexto de las teorías del desarrollo económico

\begin{tabular}{|c|c|c|}
\hline $\begin{array}{l}\text { Aspectos relevantes de la } \\
\text { competitividad }\end{array}$ & Subíndice imco & Indicador \\
\hline $\begin{array}{l}\text { Demanda agregada } \\
\mathrm{C}+\mathrm{I}+\mathrm{GP}+\mathrm{Xn} \\
\text { Impacto del comercio } \\
\text { exterior en } \mathrm{C} \text { e I } \\
\text { Intervención del Gobierno } \\
\text { como estabilizador }\end{array}$ & $\begin{array}{l}\text { Aprovechamiento } \\
\text { de las relaciones } \\
\text { internacionales }\end{array}$ & $\begin{array}{l}\text { Inversión extranjera directa, flujo de } \\
\text { pasajeros hacia y del exterior, oferta } \\
4 \text { y5 estrellas, ocupación hotelera, } \\
\text { sitios unesco, ciudad fronteriza o } \\
\text { portuaria }\end{array}$ \\
\hline $\begin{array}{l}\text { Política económica efectiva } \\
\text { Papel de los empresarios }\end{array}$ & Innovación & $\begin{array}{l}\text { Empresas, grandes empresas, } \\
\text { empresas con certificación iso } 9000 \\
\text { y } 14000 \text {, centros de investigación, } \\
\text { posgrados de calidad, patentes }\end{array}$ \\
\hline $\begin{array}{l}\text { La innovación y el } \\
\text { desarrollo tecnológico } \\
\text { La innovación científica } \\
\text { y tecnológica }\end{array}$ & $\begin{array}{l}\text { Sectores } \\
\text { precursores }\end{array}$ & $\begin{array}{l}\text { Viviendas con líneas telefónicas, } \\
\text { viviendas c/ pc, uso de Twitter como } \\
\text { proxy de uso de tecnologías de } \\
\text { información, muertes por accidentes } \\
\text { relacionados con transporte, sistema } \\
\text { de transporte masivo, red carretera } \\
\text { avanzada, aerolíneas, flujo de } \\
\text { pasajeros aéreos, líneas de autobús, } \\
\text { uso de servicios financieros, acceso } \\
\text { a servicios financieros, consumo de } \\
\text { diésel en transporte de bienes }\end{array}$ \\
\hline $\begin{array}{l}\text { Elevación del nivel de vida } \\
\text { de los ciudadanos }\end{array}$ & $\begin{array}{l}\text { Sociedad } \\
\text { incluyente } \\
\text { preparada y sana }\end{array}$ & $\begin{array}{l}\text { Espacios culturales, esr, visita a } \\
\text { museos, universidades de calidad, } \\
\text { rendimiento académico, escuelas } \\
\text { de calidad, mortalidad diabetes, } \\
\text { mortalidad infantil, médicos, } \\
\text { camas de hospital, acceso a salud, } \\
\text { crecimiento acceso a salud, viviendas } \\
\text { c/drenaje, c/ piso de tierra, viviendas } \\
\text { deshabitadas, personas bajo la línea } \\
\text { de bienestar, desigualdad en las } \\
\text { ciudades, mujeres enfuerzalaboral, } \\
\text { ingreso promedio mujer }\end{array}$ \\
\hline
\end{tabular}

Fuente: elaboración propia con base en autores revisados y el ice del imco (2015)

lida, es posible encontrar indicios de la efectividad de las medidas que han favorecido este desempeño, ya que a lo largo de los cinco años presentados en estos indicadores (2008-2012) existe un consistente desempeño elevado, manteniendo el primer lugar en "innovación”, el $2^{\circ}$ en "sectores precurso- 
res", "sociedad incluyente preparada y sana" y "aprovechamiento de las relaciones internacionales" Lo que contrasta en los periodos reportados por Cabrero 1998-2000 en el desempeño económico en $7^{\circ}$, en el institucional en $4^{\circ}$, en el urbano en el $7^{\circ}$ y en el sociodemográfico el lugar 33. El estudio de 2003-2008 de Sobrino, en que ocupa el tercer lugar y el de la Cámara de Diputados de 2008 que ubicaba al df en tres diferentes estudios en los lugares $6^{\circ}, 3^{\circ}$ y $6^{\circ}$ No deja de reconocerse que la comparación no es exacta por la discusión inicial de esta investigación referente a la concepción del problema y a la medición del mismo

Sin embargo, queda por resolver el problema de los otros seis indicadores que no han estado impulsando la competitividad, sobre los cuales habría que hacer otra revisión teórico-metodológica para identificar qué papel están ejerciendo en este índice, ya que su ponderación alcanza 52 1\% Lo que hasta este momento es posible afirmar es que existen dos indicadores que han descendido más de 14 lugares y se encuentran ubicados en los lugares 51 y 64 , correspondientes al mercado laboral y los aspectos de medio ambiente, lo que en el caso del primero deteriora el bienestar de la población y el segundo la posibilidad de un crecimiento sustentable

\section{Conclusiones}

La principal aportación de este trabajo se encuentra en la ubicación de los indicadores que en mayor medida explican el buen desempeño reciente de la competitividad regional del Distrito Federal a partir del análisis de lainformación proporcionada por el ice del imco que se encuentran en los subíndices: "sectores precursores", "sociedad incluyente preparada y sana", "aprovechamiento de las relaciones internacionales" e "innovación". Se identifica que los indicadores referidos corresponden en su gran mayoría a los propuestos por los mce en donde los determinantes de la mejora económica en un territorio son la acumulación del capital físico, capital humano y conocimientos, con énfasis en que la inclusión del conocimiento da mayor valor a los procesos de educación y a las actividades de i\&d

Si bien es posible afirmar que la teoría más divulgada y citada en las investigaciones de competitividad y en la elaboración de los más importantes índices de competitividad nacional es la de Michael Porter, en el caso de los 
estudios citados en esta investigación, referentes a la medición de la competitividad regional no presentan este marco teórico En los años recientes ha ganado importancia una nueva concepción de la competitividad que, sin dejar de dar importancia a la productividad, introduce aspectos relacionados con el conocimiento: innovación e i\&d. Así como la prioridad de lograr beneficios para la sociedad y no sólo mayores desempeños económicos

En el caso de México existe una falta de estudios longitudinales derivados de organismos con credibilidad e infraestructura suficiente para dar seguimiento y consistencia a los estudios, ya sea nacionales, regionales o de sectores específicos. Dado que en la actualidad el único organismo que ha realizado este tipo de estudios es el imco que, como se señala en el texto, se trata de una consultora independiente que realiza un gran esfuerzo por mantener esta información y que enfrenta la imposibilidad de proporcionar información oportuna dada la magnitud del problema de acceso a las fuentes pertinentes para esta labor. En los aspectos conceptuales habría que resaltar que la definición de competitividad que dirige el trabajode investigación yelaboración del Índice del imco es muy general y sin un sustento teórico robusto: "capacidad de atraer y retener talento e inversión", y que aun cuando se propongan ofrecer "elaborar propuestas viables para mejorar la capacidad de México para atraer y retener talento e inversiones", con este marco conceptual es difícil lograr una valoración sustentada en una concepción científica que confiera validez a la información. Lo que es punto de reconocimiento se refiere al adecuado sustento del modelo econométrico que permite, dentro de la salvedad anterior, dar inicio a estudios que de otra forma serían imposibles

\section{Referencias bibliográficas}

Blaug, M (1985) Economic theory in retrospect. Cambridge: Cambridge University Press Cabrero, E, Orihuela, I, y Ziccardi, A (2003) Ciudades competitivas-ciudades cooperativas: Conceptos claves y construcción de un índice para ciudades mexicanas. México: cide/ Cofemer

Centro de Estudios Sociales y de Opinión Pública (2008) La competitividad en los municipios de México. México: Cámara de Diputados lviii Legislatura

- (2012) Desarrollo regional y competitividad en México. México: Cámara de Diputados 1xi Legislatura 
Cho, D S, y Moon, H C (2002) From Adam Smith to Michael Porter. Singapur: Fulsland Offset Printing PteLtd

Huggins, R , Izushi, H, y Thompson, P(2013) "Regional Competitiveness: Theories and Methodologies for Empirical Analysis", jcc: The Business and Economics Research Journal, pp 155-172

Instituto Mexicano para la Competitividad (2015a, 25 de mayo) ¿Qué es imco? Obtenido de Conoce imco: http://imco org $\mathrm{mx}$

(2015b, 25 de mayo) Todos los índices Obtenido de imco: http://imco org mx

International Institute for Management Development (2014) Imd World Competitiveness Yearbook 2014 Results. Lausana: imd

Keynes, J(2003) Teoría general de la ocupación, el interés y el dinero. México: Fondo de Cultura Económica

Krugman, P(1994) “Competitiveness: A dangerous Obsesion”, Foreing Affairs, pp 28-44

Malecký, L (2011) “Approches to regional competitiveness evaluation in the Visegrad four countries”, Mathematil Models and Methods in Modern Science, pp 184-189

Maleki, E (2004) "Jockeyng for possition: What it means and why it matters to regional development policy when places compete", Regional Studies, pp 1101-1120 doi:http://www paca-online org/cop/docs/Malecki_-_Competition_among_regions pdf

Mattos, C A (2015) Teorías del crecimiento endógeno: Lectura desde los territorios de la periferia. Recuperado el 11 de febrero de 2015, de Estudos Avançados, 13(36): 183-208: http://www scielo br/scielo php?script=sci_arttext\&pid=S010340141999000200010\&lng=es\&nrm=iso\&tlng=es

Mun, T (1978) La riqueza de Inglaterra por el comercio exterior. México: Fondo de Cultura Económica

Porter, M (2015) Ventaja competitiva. México: Grupo Editorial Patria

Sobrino, J (2010) "Ciclos económicos y competitividad de las ciudades", en G Garza, y M C Schteingart, Desarrollo urbano y regional (pp 127-172) México: El Colegio de México

Staskevičiūtè, G., y Tamošiūnienè, R. (2010). "The Competitiveness of a Country: Evolution of the Concept", Business: Theory and Practice, pp 159-167

Thwaites Rey, M (2010) "Después de la globalización neoliberal: ¿Qué Estado en América Latina?", Observatorio Social de América Latina osal,pp 19-43

World Economic Forum (2014) The global competitiveness report 2014-2015. Ginebra: wef 